\title{
OXIDAÇÃO DE GLICEROL ASSISTIDA POR MICRO-ONDAS UTILIZANDO NANOPARTÍCULAS DE PALÁDIO SUPORTADAS EM SÍLICA COMO CATALISADOR
}

\author{
L. S. SANTOS ${ }^{1}$; B. de A. QUEIROZ ${ }^{1}$; A. JUNGES ${ }^{1}$; S. M. da S. EGUES ${ }^{1}$; E. \\ FRANCESCHI $^{1}$; C. DARIVA ${ }^{1}$; A. F. SANTOS ${ }^{1}$; M. F. HEREDIA ${ }^{1}$ \\ ${ }^{1}$ Universidade Tiradentes, Instituto de Tecnologia e Pesquisa/Itp, Aracaju-SE \\ E-mail para contato: leonardo-se@hotmail.com
}

\begin{abstract}
RESUMO - O uso de nanopartículas metálicas como catalisadores vem sendo foco de estudos por apresentarem resultados significativos em reações químicas. Devido ao excesso de glicerol produzido pela transesterificação do biodiesel, faz-se necessário o desenvolvimento de processos alternativos de transformação desta matéria prima a fim de formar produtos de alto valor agregado como os ácidos oxálico, glicólico, fórmico, acético e demais. Assim, o objetivo deste trabalho é propor uma reação de oxidação de glicerol utilizando catalisador $\mathrm{Pd} / \mathrm{SiO}_{2}$ com baixo teor de metal $(0,5 \%$ wt $\mathrm{Pd})$ e peróxido de hidrogênio $\left(\mathrm{H}_{2} \mathrm{O}_{2}\right)$ como agente oxidante em reator batelada assistido por micro-ondas monomodo. A utilização de irradiação micro-ondas causa um aumento nas taxas de conversões do glicerol em um curto tempo de reação. Foram avaliados os efeitos de temperatura $\left(60,80\right.$ e $\left.100{ }^{\circ} \mathrm{C}\right)$, potência de irradiação das micro-ondas $(100$, 200 e $300 \mathrm{~W})$ e massa de catalisador $(0,110,15$ e $0,22 \mathrm{~g})$. Os resultados indicaram que foi possível converter até $60 \%$ do glicerol em 15 min usando $0,22 \mathrm{~g}$ de catalisador, $300 \mathrm{~W}$ de potência e temperatura de $100{ }^{\circ} \mathrm{C}$, com seletividade alta para o gliceraldeído e ácido oxálico.
\end{abstract}

\section{INTRODUÇÃ̃}

A pesquisa e o desenvolvimento que têm como objetivos encontrar fontes de energias alternativas ao petróleo está em franca evolução, buscando assim minimizar os problemas ambientais que os combustíveis derivados do petróleo vêm causando. Várias são as fontes de energia alternativa que se tem buscado, tais como os biocombustíveis. Neste cenário o biodiesel se destaca como fonte de geração de energia.

Durante o processo de produção do biodiesel o principal subproduto gerado é o glicerol. O glicerol é um subproduto da reação de transesterificação que na forma pura possui diversas utilidades na cadeia industrial, podendo ser aplicado como aditivo e também utilizado em indústrias farmacêuticas e químicas (Rivaldi et al., 2012). O glicerol apresenta total solubilidade em água e álcoois em geral como também em éter, mas possui insolubilidade nos hidrocarbonetos. Esta solubilidade em água se dá pela presença das três hidroxilas como observado na Figura 1 (Gupta et al., 2012). 


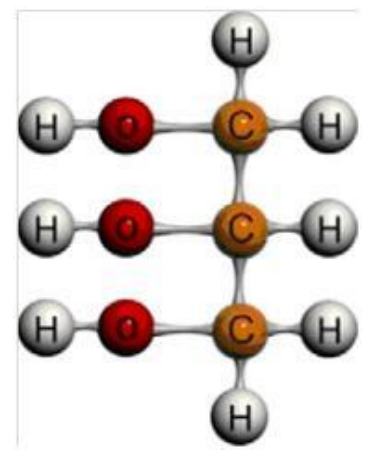

Figura 1 - Estrutura molecular do glicerol (RAHMAT et al, 2010).

Assim, com o aumento do consumo de biodiesel no cenário internacional, a oferta de glicerol no mercado supera em demasia a sua demanda, gerando assim uma queda considerável no seu valor comercial (Leoneti et al., 2012). Diversas são as aplicações para o glicerol. Porém, devido ao grande excedente deste produto no mercado, o desenvolvimento de processos alternativos de transformação desta matéria prima se faz necessário. Dentre estes processos, a oxidação química é a responsável por transformar esta matéria prima em produtos de alto valor agregado com intensa aplicação na química fina, proporcionando um fim mais nobre para o resíduo da produção de biodiesel (Kamonsuangkasem et al., 2011).

A oxidação de glicerol é um processo reacional complexo, dependendo do catalisador utilizado e do meio em que a reação ocorre, pelo simples fato do grande número de produtos que são formados durante a reação, dentre os quais se destacam o acido glicérico e a dihidroxacetona (Behr et al., 2008). Por isso, a escolha de um bom catalisador associado a um meio reacional que proporcione controlar a seletividade na formação de produto é de grande interesse (Nunes et al., 2013; Diaz et al., 2014).

A aplicação das micro-ondas vem avançando em busca de reduções significativas no tempo de reação, aumento da produtividade e diminuição dos produtos secundários. Assim uma das técnicas utilizadas é a ativação cíclica por micro-ondas, e segundo a literatura, em baixa potência é gerada uma ativação moderada da mistura reacional e consequentemente uma redução no tempo de reação. A técnica baseia-se no aquecimento repetido da mistura da reação e seu arrefecimento em curtos ciclos, utilizando uma menor energia de irradiação (Paakkonen et al., 2010).

\section{MATERIAIS E MÉTODOS}

Nesta seção serão detalhados todos os procedimentos necessários para realização dos experimentos de oxidação do glicerol via micro-ondas. Utilizando padrões de ácido glicólico, ácido oxálico, glicerol, ácido tartrônico, ácido fórmico, ácido acético, gliceraldeído, dihirdroxiacetona, como fase móvel para análise cromatográfica utilizou-se o ácido sulfúrico (97\% de pureza). Gases foram aplicados na caracterização do catalisador. 


\subsection{Catalisador $\mathrm{Pd} / \mathrm{SiO}_{2}$}

Neste trabalho foi utilizado um catalisador de paládio suportado em sílica $\left(\mathrm{Pd} / \mathrm{SiO}_{2}\right)$ preparado e caracterizado previamente por Junges (2011). O catalisador foi sintetizado por impregnação úmida com uma solução aquosa de $\mathrm{Pd}\left(\mathrm{NO}_{3}\right)_{2} \cdot 2 \mathrm{H}_{2} \mathrm{O}$, com teor nominal de paládio de 0,5\% em massa. Foram realizadas diversas caracterizações do catalisador como análise química, medida de área superficial específica, área metálica, dispersão e tamanho da partícula como mostra a Tabela 1. Foi realizada caracterização adicional de redução à temperatura programada (TPR - do inglês: Temperature-programmed reduction).

Tabela 1- Características do catalisador $\mathrm{Pd} / \mathrm{SiO}_{2}$ utilizado nas reações de oxidação do glicerol (Junges 2011).

\begin{tabular}{|c|c|c|c|c|c|c|}
\hline Cat & $\begin{array}{l}\text { Composiçã } \\
\text { o de Pd }^{a} \\
(\% \text { massa })\end{array}$ & $\begin{array}{c}\text { Área } \\
\text { Superficial }^{b} \\
\left(\mathrm{~m}^{2} / \mathrm{g}_{\text {cat }}\right)\end{array}$ & $\begin{array}{c}\text { Tamanho } \\
\text { do poro } \\
\text { (ঐ) }\end{array}$ & $\begin{array}{c}\text { Área } \\
\text { Metálica } \\
\left(\mathbf{m}^{2} / \mathbf{g}_{\text {cat }}\right)\end{array}$ & $\begin{array}{c}\text { Dispersão }^{c} \\
(\%)\end{array}$ & $\begin{array}{c}\text { Tamanho } \\
\text { partícula } \\
(\text { nm) }\end{array}$ \\
\hline $0,5 \% \mathrm{Pd} / \mathrm{SiO}_{2}$ & 0,579 & 314 & 150 & 0,4422 & 15,7807 & 7,10 \\
\hline
\end{tabular}

${ }^{\mathrm{a}}$ Espectrometria de plasma indutivamente acoplado; ${ }^{\mathrm{b}}$ Adsorção e dessorção de $\mathrm{N}_{2}$ a $77 \mathrm{~K}$; ${ }^{\mathrm{c}}$ Adsorção química de hidrogênio.

\subsection{Aparato experimental}

Os experimentos via micro-ondas monomodo foram realizados em um reator CEM-Discover representado na Figura 2. A potência máxima aplicada foi de $300 \mathrm{~W}$, com temperatura máxima de $100{ }^{\circ} \mathrm{C}$, o equipamento emite radiação a uma frequência de $2,45 \mathrm{Ghz}$. O volume reacional foi de $20 \mathrm{ml}$ para cada reação.

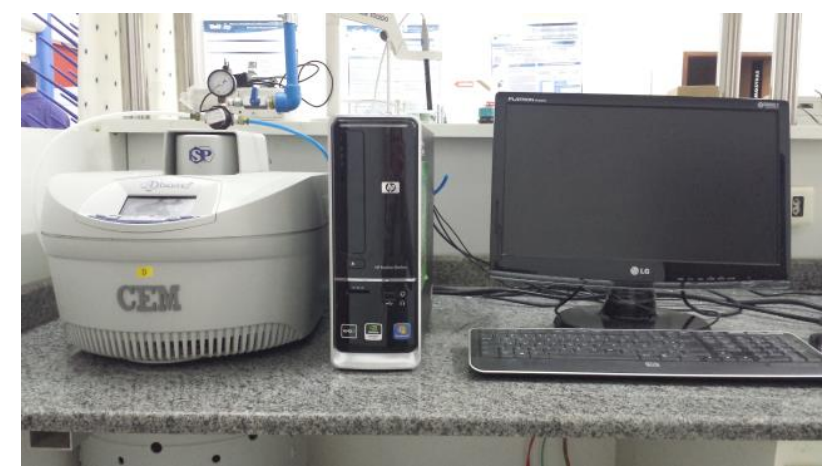

Figura 2- Unidade experimental com reator CEM-Discover.

As variáveis, potência, temperatura e agitação foram controladas por um sistema de controle interno. 


\subsection{Reação de oxidação de glicerol}

Primeiramente as soluções para as reações foram preparadas com uma quantidade de $3 \mathrm{M}$ de glicerol (Chornaja et al., 2013) e $4 \mathrm{M}$ de $\mathrm{H}_{2} \mathrm{O}_{2}$ (Sankar et al., 2009). Uma solução de glicerol foi preparada em um balão de $20 \mathrm{ml}$ na presença de água como solvente e massa do glicerol de $0,55 \mathrm{~g}$, nessa mesma solução foi diluída $2,7 \mathrm{~g}$ de peróxido de hidrogênio. Em seguida o catalisador reduzido foi colocado ao reator de quartzo e misturado com a solução de glicerol e peróxido de hidrogênio. As massas de catalisador utilizadas foram de 0,11, 0,15 e 0,22 g. As temperaturas foram: 60,80 e $100{ }^{\circ} \mathrm{C}$, potência: 100,200 e $300 \mathrm{~W}$, e o tempo de reação de 15 minutos. . As reações ocorreram no tempo de 15 minutos com agitação constante. Após o término da reação, o reator é resfriado através de corrente de convecção de ar comprimido até atingir a temperatura de $40{ }^{\circ} \mathrm{C}$ e redução de pressão até pressão atmosférica.

As amostras das reações foram analisadas por cromatografia líquida de alta eficiência (CLAE) num cromatógrafo UFLC Shimadzu, com detector UV-Vis SPD-20A. O comprimento de onda selecionado foi de $210 \mathrm{~nm}$ (Hu et al., 2011) por representar melhor identificação dos picos em relação os comprimentos de onda 190 e $245 \mathrm{~nm}$ que também foram analisados. A coluna de separação utilizada foi uma coluna de troca iônica (SUPELCOGEL C-610H) de $30 \mathrm{~cm}$ de comprimento e $7,8 \mathrm{~mm}$ de diâmetro interno. Uma pré-coluna (CARBO-H 4x30mm ID) foi conectada a coluna para evitar a contaminação e entupimento da coluna com impurezas. A fase móvel utilizada foi uma solução de $\mathrm{H}_{2} \mathrm{SO}_{4}$ na concentração de $0,001 \mathrm{M}$ e fluxo de $0,6 \mathrm{ml} / \mathrm{min}$ ) (Rodrigues et al., 2011; Prieto et al., 2012). Todas as amostras estudas foram injetadas em um volume $20 \mu \mathrm{l}$, com tempo de análise de 25 minutos.

A quantificação dos produtos da reação e do glicerol não reagido foi obtida através de dados de área dos picos gerados nos cromatogramas e da equação da curva de calibração. A conversão total do glicerol foi determinada pela Equação 1. A seletividade é uma propriedade ligada ao catalisador devido à variedade de reações possíveis. A seletividade dos produtos obtidos foi calculada a partir da Equação 2 e 3 (Nie et al., 2012).

$$
C(\%)=\frac{\text { concentração inicial do glicerol }- \text { concentração final do glicerol }}{\text { concentração inicial do glicerol }} * 100
$$

\section{$\operatorname{Si}(\%)$}

mols do produto da reação * átomos de carbono prod.formado

$=\overline{\text { mols inicial do glicerol - mols do glicerol no final da reação } * \text { átomos de carbono prod.formado }}$ $* 100$

$$
S_{\text {normalizada }}(\%)=\frac{\text { Seletividade }(\%) * 100}{\text { Rendimento }}
$$




\section{RESULTADOS E DISCUSSÃO}

\subsection{Análise cromatográfica}

A análise cromatográfica obteve diferentes resultados para cada parâmetro estudado. Topos os produtos da reação foram quantificados para verificar a conversão de glicerol e os produtos com maior concentração em diferentes condições. A Figura 3 mostra um cromatograma típico de um padrão de glicerol utilizando ácido sulfúrico como fase móvel com fluxo de $0,6 \mathrm{ml} / \mathrm{min}$.

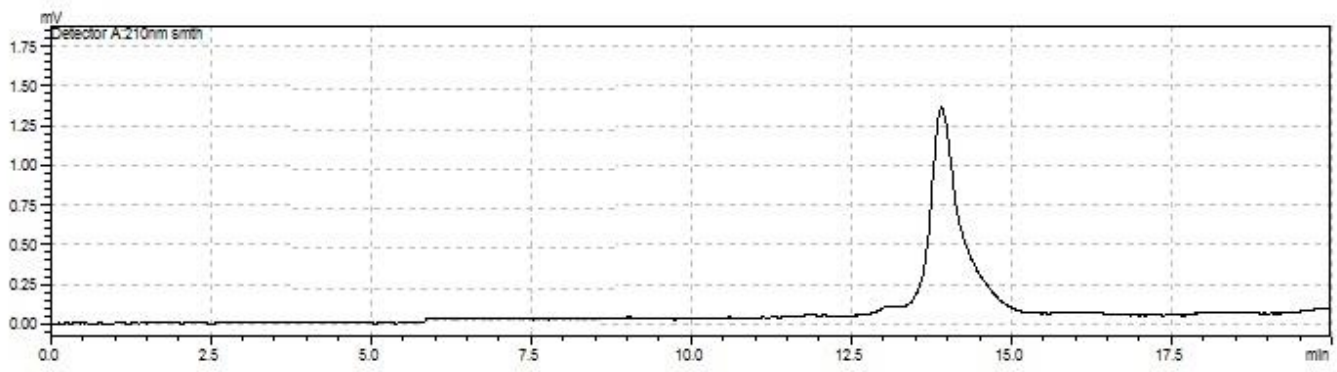

Figura 3 - Cromatograma da solução padrão de glicerol.

\subsection{Reação de oxidação do glicerol}

A reação de oxidação foi realizada em diferentes temperaturas $\left(60,80\right.$ e $\left.100{ }^{\circ} \mathrm{C}\right)$, potência $(100,200$ e $300 \mathrm{~W})$ e massa de catalisador $(0,110,15$ e $0,22 \mathrm{~g})$ a fim de se observar a conversão do glicerol. A reação em micro-ondas se mostrou bastante satisfatória por apresentar conversões de 40 a $60 \%$ em um tempo de 15 minutos conforme a Tabela 2.

Tabela 2- Conversão de glicerol e seletividade dos produtos na oxidação de glicerol por $\mathrm{Pd} / \mathrm{SiO}_{2}$ como catalisador, no tempo de 15 minutos.

\begin{tabular}{cccccc}
\hline Temp. $\left({ }^{\circ} \mathbf{C}\right)$ & $\begin{array}{c}\text { Potência } \\
(\text { Watts })\end{array}$ & $\begin{array}{c}\text { Massa de } \\
\text { catalisador } \\
(\mathbf{g})\end{array}$ & $\begin{array}{c}\text { Conversão do } \\
\text { glicerol } \\
(\boldsymbol{\%})\end{array}$ & $\begin{array}{c}\text { Gliceraldeído } \\
(\boldsymbol{\%})\end{array}$ & $\begin{array}{c}\text { Ácido Oxálico } \\
(\boldsymbol{\%})\end{array}$ \\
\hline $\mathbf{6 0}$ & 300 & 0,22 & 60 & 49,7 & 47,0 \\
$\mathbf{6 0}$ & 100 & 0,11 & 53 & 37,3 & 57,7 \\
$\mathbf{1 0 0}$ & 100 & 0,22 & 49 & 79,7 & 16,1 \\
$\mathbf{1 0 0}$ & 300 & 0,22 & 40 & 91,3 & 7,0 \\
$\mathbf{8 0}$ & 200 & 0,15 & 51 & 69,1 & 29,3 \\
\hline
\end{tabular}


O catalisador a base de nanopartículas de paládio suportado em sílica $\left(\mathrm{Pd} / \mathrm{SiO}_{2}\right)$ mostrou uma conversão para o glicerol de $60 \%$. Essa conversão foi obtida empregando temperatura de 60 ${ }^{\circ} \mathrm{C}$, com potência de $300 \mathrm{~W}$ e massa de catalisador de $0,22 \mathrm{~g}$. Um estudo de oxidação de glicerol foi realizado por Luque et al., (2008) verificando que ao utilizar um catalisador com maior concentração de metal a conversão do glicerol varia entre 58 e $98 \%$ com a mesma condição de potência, temperatura e massa de catalisador aplicadas neste trabalho.

A maior seletividade foi obtida para o gliceraldeído com $91,4 \%$ aplicando uma potência de $300 \mathrm{~W}$, temperatura de $100{ }^{\circ} \mathrm{C}$ e conversão de $49 \%$ com massa de catalisador em $0,11 \mathrm{~g}$. Ao utilizar a mesma potência só que uma quantidade de catalisador maior $0,22 \mathrm{~g}$ a seletividade permaneceu quase que a mesma $91,3 \%$, ou seja, a concentração de catalisador nesse experimento não influencia a formação desse produto.

A utilização do peróxido de hidrogênio como agente oxidante se dá pelo baixo custo e vasta formação de produtos em reações de oxidação do glicerol. Vale ressaltar que um aumento no $\mathrm{H}_{2} \mathrm{O}_{2}$ no sistema não varia significativamente a atividade e seletividade do catalisador durante a reação (Luque et al., 2008). Em altas temperaturas ocorre a decomposição do peróxido de hidrogênio o que afeta a conversão e seletividade de alguns produtos como o dihidroxiacetona, ácido glicólico. A desestabilização da estrutura do peróxido em altas temperaturas desacelera a quebra do glicerol diminuindo sua conversão como observado na Tabela 2 ao aplicar uma temperatura de $100{ }^{\circ} \mathrm{C}$ a conversão tem um decréscimo para $40 \%$ em relação a temperatura de $60{ }^{\circ} \mathrm{C}$ que a conversão chega a $60 \%$.

\subsection{Cinética de reação de oxidação do glicerol}

Uma cinética de reação foi construída para observar o comportamento do consumo de glicerol em relação ao tempo. O estudo cinético foi realizado em diferentes tempos, 0, 0,5, 1, 2, 5, $8,10,15,20$ e 30 minutos. Foi utilizada uma solução com 0,55 g de glicerol diluído em $20 \mathrm{ml}$ de água, obtendo uma concentração de glicerol de $27 \mathrm{~g} / \mathrm{L}$. Foi introduzida a esta solução $2,7 \mathrm{~g}$ de peróxido de hidrogênio e $0,11 \mathrm{~g}$ de catalisador reduzido. As condições empregadas foram potência de $300 \mathrm{~W}$, temperatura de $100{ }^{\circ} \mathrm{C}$ e alta agitação no reator micro-ondas monomodo CEMDiscover.

Os resultados mostraram uma conversão de $50 \%$ de glicerol e alta seletividade para o gliceraldeído e ácido oxálico. O consumo de glicerol é significativo até 10 minutos de reação, permanecendo quase que constante até 30 minutos como mostra a Figura 4. 


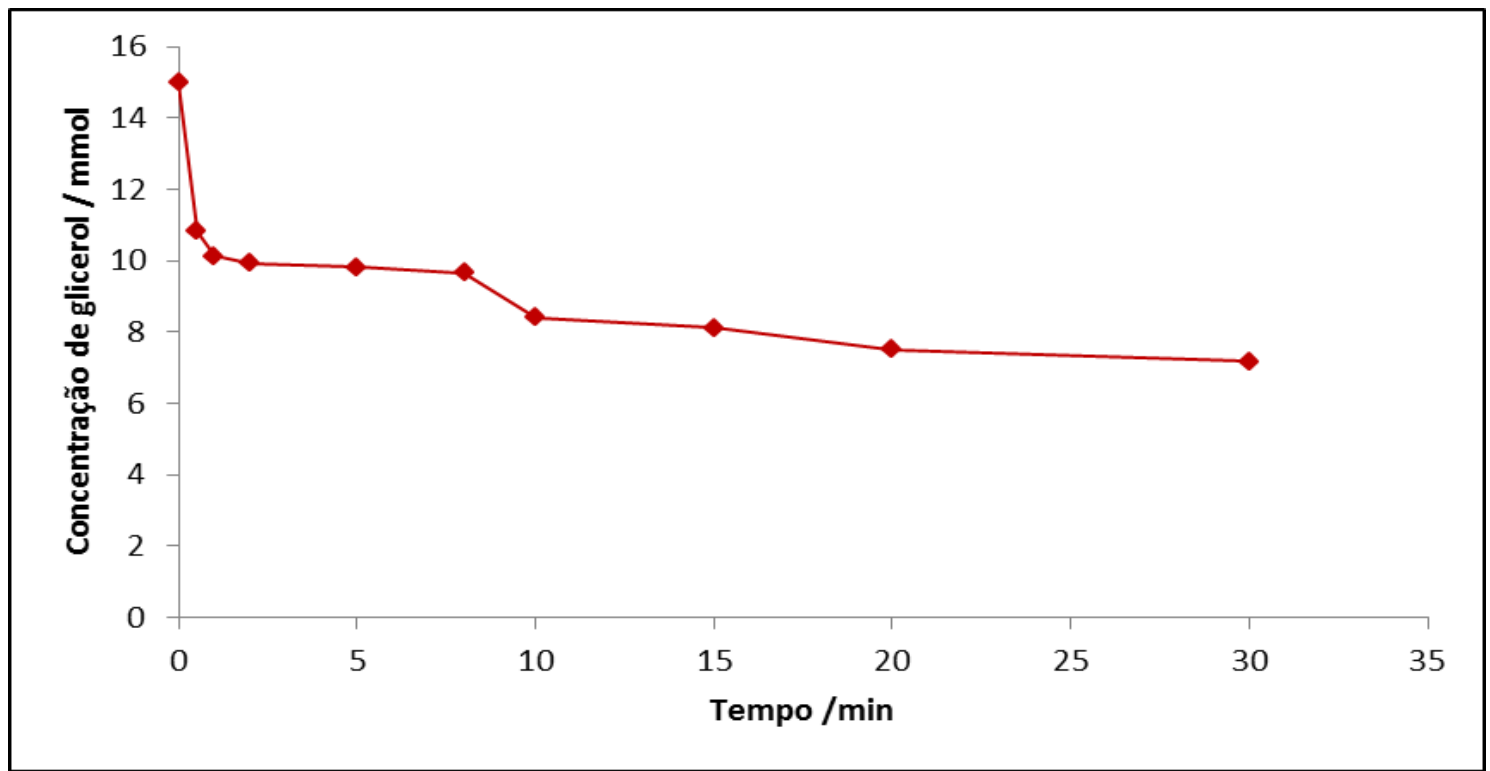

Figura 4 - Cinética de oxidação de glicerol em 0,5\% $\mathrm{Pd} / \mathrm{SiO}_{2}$. Condições reacionais: $3 \mathrm{M}$ glicerol, $300 \mathrm{~W}, 100{ }^{\circ} \mathrm{C}, 20 \mathrm{ml}, 0,11 \mathrm{~g}$ de catalisador, Glicerol $/ \mathrm{H}_{2} \mathrm{O}_{2}=4$.

A reação era realizada uma por vez, assim a amostra só era recolhida no final da reação. Cada tempo de análise possui uma reação independente já que o sistema impossibilita a retirada de amostragem durante a realização da reação. A elaboração de uma cinética de glicerol é bastante complexa devido a versatilidade desse co-produto em formar diferentes produtos.

\section{REFERÊNCIAS}

BEHR，A.; EILTING，J.; IRAWADI，K.; LESCHINSKI，J.; LINDNER，F. Improved utilisation of renewable resources: New important derivatives of glycerol. Green Chemistry. Vol. 10. p. 1-140, 2008.

CHORNAJA, S.; DUBENCOV, K.; KAMPARS, V.; STEPANOVA, O.; ZHIZHKUN, S.; SERGA, V.; KULIKOVA, L. Oxidation of glycerol with oxygen in alkaline aqueous solutions in the presence of supported palladium catalysts prepared by the extractive-pyrolytic method. Reac Kinet Mech Cat, 2012.

GUPTA, M.; KUMAR, N. Scope and opportunities of using glycerol as an energy source. Renewable and Sustainable Energy Reviews. p. 4551-4556, 2012.

HU, W. LOWRY, B.; VARMA, A.; Kinetic study of glycerol oxidation network over Pt-Bi/C catalyst. Applied Catalysis B: Environmental 106. p. 123-132, 2011.

JUNGES, Alexander. Estudo da Síntese de Nanopartículas Metálicas de Paládio para uso em Catálise Heterogênea. Tese de Mestrado, UFSM, Santa Maria, RS, Brasil, 2011. 
KAMONSUANGKASEM, K.; THERDTHIANWONG, S.; THERDTHIANWONG, A Hydrogen Production via Oxidative Steam Reforming of Biodiesel By-products over $\mathrm{Ni} / \mathrm{CeO} 2-\mathrm{ZrO} 2 / \mathrm{Al}_{2} \mathrm{O}_{3}$ Catalyst. International Conference on Chemistry and Chemical Process, 2011.

LEONETI, A. B; LEONETI, V. A.; VALLE, S.; OLIVEIRA, W. B. Glycerol as a by-product of biodiesel production in Brazil: Alternatives for the use of unrefined glycerol. Renewable Energy 45, p.138-145, 2012.

LUQUE, R.; BUDARIN, V.; CLARK, J. H.; MACQUARRIE, D. J. Glycerol transformations on polysaccharide derived mesoporous materials. Applied Catalysis B: Environmental. p. 157-162, 2008.

NIE R.; LIANG, D.; SHEN, L.; GAO, J.; CHEN, P.; HOU, Z. Selective oxidation of glycerol with oxygen in base-free solution over MWCNTs supported PtSb alloy nanoparticles. Applied Catalysis B: Environmental 127, p. 212- 220, 2012.

NORONHA F.B, F. B; D. A. G. ARANDA; A.P. ORDINE; M.SCHMAL. Catalysis Today, 57, p. 275, 2000.

NUNES, C. A.; GUERREIRO, M. C. Chemometric approaches on glycerol oxidation with $\mathrm{H}_{2} \mathrm{O}_{2}$ over supported gold nanoparticles. Journal of Molecular Catalysis A: Chemical 370, p.145-151, 2013.

PÄÄKKÖNEN, S.; PURSIAINEN, J.; LAJUNEN, M. Fast oxidation of secondary alcohols by the bromate-bromide system using cyclic microwave heating in acidic water. Tetrahedron Letters, v. 51, p.6695-6699, 2010.

PRIETO, J. C. B.; PECHA, J.; KAŠPÁRKOVÁ, V.; KOLOMAZNÍK, K. Determination of glycerol derivatives by High-performance liquid chromatography. Advances in Environment, Biotechnology and Biomedicine. 2012.

RIVALDI, J. D.; SARROUH, B. F.; BRANCO, R. F.; MANCILHA, I. M.;SILVA, S. S. Biotechnological Utilization of Biodiesel-Derived Glycerol for the Production of Ribonucleotides and Microbial Biomass. Appl Biochem Biotechnol. p. 2054-2067, 2012.

RODRIGUES, E. G.; CARABINEIRO, S. A. C; CHEN, X.; DELGADO, J. J.; FIGUEIREDO, J. L.; PEREIRA, M. F. R.; ÓRFÃO, J .J. M. Selective Oxidation of Glycerol Catalyzed by Rh/Activated Carbon: Importance of Support Surface Chemistry. Catal Lett. p. 420-431, 2011.

SANKAR. M. et. al. Oxidation of Glycerol to Glycolate by using Supported Gold and Palladium Nanoparticles. ChemSusChem. p. 1-8, 2009. 